\title{
Maternal Consumption of Lactobacillus GG During Pregnancy Prevents Infant Eczema at 1 Year of Age
} \author{
Samuli Rautava ${ }^{4}$ and Seppo Salminen ${ }^{4}$ \\ ${ }^{1}$ Department of Pediatrics, Toho University School of Medicine, 6-11-1, Omori-Nishi, Ota-ku, Tokyo 143-8541, Japan \\ ${ }^{2}$ Uchino Obstetrics and Gynecology and Pediatrics, 1-8-6, Higashi, Kunitachi-shi, Tokyo 186-0002, Japan \\ ${ }^{3}$ Takanashi Milk Products Co., Ltd, 5 Honjuku-cho, Asahi-ku, Yokohama, 241-0023, Japan \\ ${ }^{4}$ Department of Food Development, University of Turku, FI-20014 TURUN YLIPISTO, Finland
}

Yumiko Komine ${ }^{1,2 *}$, Misa Watanabe ${ }^{1}$, Takehiko Soutome ${ }^{1}$, Takako Uchino², Mamiko Dobashi², Gaku Harata ${ }^{3,4}$ Kenji Miyazawa ${ }^{3}$, Fang He

\begin{abstract}
Objective: We investigated the effects of probiotic yogurt administration to mothers during late pregnancy on intestinal microbiota and the incidence rate of atopic eczema in infants at one year of age.

Methods: Eighteen pregnant women were given $100 \mathrm{~g}$ of yogurt containing Lactobacillus rhamnosus $G \mathrm{G}$ (LGG), from day 1 of the $33^{\text {rd }}$ week of gestation until parturition (LGG group), and the results obtained from their newborns were compared to those from infants born to 14 mothers who had not received this probiotic treatment (Control group). Lactobacillus GG bacteria (LGG bacteria) in infants' feces at 4 and 6 months of age were compared using quantitative polymerase chain reaction. The relationship between the detection rate of LGG bacteria and the presence or absence of eczema at the age of one year was evaluated.

Results: The LGG bacteria detection rate at 4 months was significantly higher in the LGG than in control group $(p<0.05)$, with a lower incidence rate of eczema in the LGG than in the control group $(p<0.05)$. Among infants with eczema at 4 and 6 months, the rate of eczema clearing at 1 year was higher for the LGG than the control group $(p<0.05)$. Administering LGG to pregnant women was effective in lower the incidence of eczema at 1 year, regardless of the LGG bacteria in infants $(p<0.03)$.
\end{abstract}

Conclusion: Giving LGG yogurt probiotics to pregnant women during late pregnancy is useful in preventing eczema in infants at 1 year of age.

Keywords: Eczema; Intestinal flora; Lactobacillus GG yogurt; Lactobacillus rhamnosus GG; Prenatal fecal bifidobacterial microflora; Probiotics prevention

\section{Introduction}

The effect of probiotics in preventing the development of allergies has been reported since 2001 [1]. A meta-analysis published in 2015 provided evidence of the efficacy of probiotics, administered to pregnant and lactating mothers, in preventing atopic disease and allergies in infants [2-4]. The World Allergy Organization-McMaster University Guidelines for Allergic Disease Prevention (GLAD-P) determined that there is likely a net benefit of using probiotics for the prevention of eczema in infants, when the probiotics are used by [5], a) pregnant women at high risk for having an allergic child; b) women who are breastfeeding infants at high risk of developing an allergy; and c) infants at high risk of developing an allergy. However, the evidence underlying these recommendations is not very strong. Therefore, we have been conducting an intervention study, since 2009, in which Lactobacillus rhamnosus GG (LGG) yogurt is given to pregnant women during late pregnancy and we monitor the health of their infants' skin and their intestinal microbiota. As part of this ongoing study, we have previously reported that exposure of mothers to LGG during the last trimester of pregnancy increased the intestinal bifidobacteria in infants, which is believed to be useful in preventing atopic eczema [6]. In particular, the detection rate of Bifidobacterium, the number of bacteria, the number of bifidobacterial species, and the detection rate of Bifidobacterium breve ( $B$. breve) were higher among infants whose mother consumed LGG during the last trimester of pregnancy than in those whose mother were not exposed to LGG, with this difference being specifically greater between 4 and 9 months after birth.
In the current study, our aim was to evaluate the influence of probiotic administration to pregnant women on the intestinal microbiota of infants after weaning (at 4 and 6 months) and to evaluate the relationship between intestinal microbiota and the prevalence of eczema at 1 year of age.

\section{Materials and Methods}

\section{Participants}

Participants were recruited from among the 1714 pregnant women who made regular visits to our obstetrics department, from March 2009 through April 2015, and who planned to deliver their baby at our hospital. Since it is ethically difficult to randomly assign participants to the probiotic intervention group, we recruited a probiotic administration group and a control group for every month. Before participating in the study, all mothers received a full and clear description of the study goals and procedures and provided their written informed consent. All study procedures were completed in accordance with the Helsinki Declaration and were reviewed and approved by the Toho University Medical Ethics

*Corresponding author: Yumiko Komine, Department of Pediatrics, Toho University School of Medicine, 6-11-1, Omori-Nishi, Ota-ku, Tokyo 143-8541, Japan, Phone: 703 3762-4151; Fax: 703-3298-8217; E-mail: ucchi-no@med.toho-u.ac.jp

Received May 14, 2018; Accepted May 17, 2018; Published May 21, 2018

Citation: Komine Y, Watanabe M, Soutome T, Uchino T, Dobashi M, et al. (2018) Maternal Consumption of Lactobacillus GG During Pregnancy Prevents Infant Eczema at 1 Year of Age. J Allergy Ther 9: 277. doi:10.4172/2155-6121.1000277

Copyright: $\odot 2018$ Komine $Y$, et al. This is an open-access article distributed under the terms of the Creative Commons Attribution License, which permits unrestricted use, distribution, and reproduction in any medium, provided the original author and source are credited. 
Committee (approval no. 20012). The study was registered in the UMINCTR Clinical Trial Registry (UMIN 000017255).

\section{Exclusion criteria}

Women with pre-eclampsia, pregnancy-induced hypertension, gestational diabetes, allergies to milk products, or infectious diseases were excluded from the study.

\section{Design}

Our study group included 90 cases in the LGG group and 68 in the control group. Infants were breastfed for at least 1 month after birth, and those who did not require antimicrobial oral medicine or topical steroid medicine up to the age of 6 months and who were followed-up to 1 year of age for eczema screening were included in the analysis. From this group, we excluded cases in which the mother was administered more than the usual dose of antibiotics, before and after parturition, where usual care in our hospital includes the provision of antibiotics to mothers within 3 days after parturition. Based on these criteria, 18 cases in the LGG group and 14 cases in the control group were included in the final analysis.

The occurrence of eczema was determined using a parentreport questionnaire. Using the severity scoring of atopic dermatitis (SCORAD) questionnaire [7], we asked mothers to describe the site and nature of their infant's eczema on the doll figure provided in the questionnaire. Eczema was generally defined by the presence of papules, erosions, cracks in the ears, and/or itching.

The 18 women in the LGG group started the probiotic regimen on the first day of the $33^{\text {rd }}$ week of gestation. The regimen consisted of consuming one 100-g package of yogurt containing LGG per day until parturition, with a daily record of consumption maintained by each mother. Therefore, mothers had a 1 to 2 month exposure to LGG. The LGG yogurt (brand name "Onaka e GG") was kindly supplied by the Takanashi Milk Products Co., Ltd. (Yokohama, Japan). Their own research confirmed that each 100-g serving of yogurt, which is the recommended daily amount, contains $>1.4 \times 10^{10}$ colony-forming units (CFUs) of LGG. Mothers in the control group did not receive this probiotic regimen during late gestation. Other than the prescribed LGG, mothers in both groups were instructed to consume as little fermented foods, such as natto (fermented soybeans) and yogurt, as possible.

Fecal samples were collected from infants at 4 and 6 months after birth for between-group comparison. Samples were assigned a random number to ensure anonymity in the blinded analysis. The stool samples were frozen at $-20^{\circ} \mathrm{C}$ after collection at home and all samples were stored at $-80^{\circ} \mathrm{C}$ until DNA extraction and quantitative polymerase chain reaction (Q-PCR), which were performed at Turku University.

\section{DNA-extraction}

DNA was extracted from $200 \mathrm{mg}$ stool samples using the ISOFECAL for Beads Beating kit (Nippon Gene Co., Ltd., Japan) and the Mini Bead Beater kit (Biospec Products, Inc., USA), based on manufacturers' instructions [8].

\section{Quantitative PCRs}

Quantitative PCR was performed as previously described $[9,10]$. PCR amplification and detection were performed using an ABI PRISM 7300-PCR sequence detection system (Applied Biosystems, Foster City, CA). The bacterial concentration in each sample was determined by comparing the $\mathrm{Ct}$ values obtained from standard curves formed from serial dilutions of DNA extracted from a known amount of LGG cells.

\section{Data collection}

The background characteristics of mothers and newborns were retrospectively analyzed from their hospital records. The following information from mothers was extracted for analysis: age, history of allergies, complications during pregnancy, use of antibiotics during pregnancy, turbidity of amniotic fluid, and parturition status. Information on newborns included: sex, gestational weeks, type of delivery, height and weight at birth, and any complications before or around the time of delivery.

\section{Statistical analysis}

Student's t-test and chi-squared $(\chi 2)$ analyses were used to evaluate between-group differences in the characteristics of mothers and newborns, either at or soon after delivery. Between-group differences in the prevalence of LGG and eczema were evaluated using $\chi 2$ analysis, as well as Fisher's direct probability calculation method and logistic regression analysis. All analyses were performed using SPSS for Windows (version 18; SPSS Inc., Chicago, IL, USA).

\section{Results}

\section{Background information on mothers and newborns}

There were no differences in the characteristics of the mothers between the LGG and control groups with respect to age, history of allergies, rate of cesarean delivery, intake of milk products after delivery, or complications during pregnancy. For newborns, there were no between-group differences with regard to age, sex ratio, weeks of gestation, birth weight and height, and the introduction to baby food at 6 months of age (Table 1).

\section{Detection rate of LGG bacteria}

As shown in Figure 1, the rate of LGG bacteria detection at 4 months of age was significantly higher in the LGG than the control group ( $\mathrm{p}=0.02$ ), with no significant between-group difference at 6 months of age.

\section{Prevalence of Eczema at 1 year of age}

As shown in Figure 2, the prevalence of eczema was significantly lower in the LGG $(16.7 \%)$ than the control $(57.1 \%)$ group $(\mathrm{p}=0.02)$.

\section{Relationship between the incidence of eczema (at 4 and 6 months) and eczema at 1 year of age}

Among infants with eczema at 6 months of age, eczema cleared in $77.8 \%$ of cases at 1 year of age in the LGG group, compared to only $28.6 \%$ in the control group (Figure 3 ).

\begin{tabular}{|c|c|c|c|c|}
\hline & & LGG & Control & $P$ value \\
\hline \multirow{4}{*}{ M } & Age (years) & $31.2 \pm 4.53$ & $31.8 \pm 5.12$ & n.s. \\
\hline & Allergy $* \%)$ & 61.1 & 57.1 & n.s. \\
\hline & Cesarean delivery & 0 & 0 & n.s. \\
\hline & $\begin{array}{l}\text { Intake of milk products } \\
\text { after delivery }\end{array}$ & 9 & 7 & n.s. \\
\hline \multirow{6}{*}{$\mathbf{N}$} & Male:Female & $12: 6$ & $5: 9$ & n.s. \\
\hline & Gestational weeks & $39 w 0 d$ & $40 w 1 d$ & n.s. \\
\hline & Height (cm) & $49.0 \pm 1.54$ & $48.8 \pm 1.45$ & n.s. \\
\hline & Weight (g) & $3050.1 \pm 296.3$ & $3114.4 \pm 359.9$ & n.s. \\
\hline & Baby food begins (6 mo) & 14 & 11 & n.s. \\
\hline & Breast:Mix:Milk (6 mo) & $12: 6: 0$ & $8: 4: 2$ & n.s. \\
\hline
\end{tabular}

Table 1: Background information on mothers and newborns; M-Mothers N-Newborns; *-Bronchial asthma; atopic dermatitis; allergic rhinitis; food allergy; mo- months. 
Citation: Komine Y, Watanabe M, Soutome T, Uchino T, Dobashi M, et al. (2018) Maternal Consumption of Lactobacillus GG During Pregnancy Prevents Infant Eczema at 1 Year of Age. J Allergy Ther 9: 277. doi:10.4172/2155-6121.1000277

Page 3 of 5

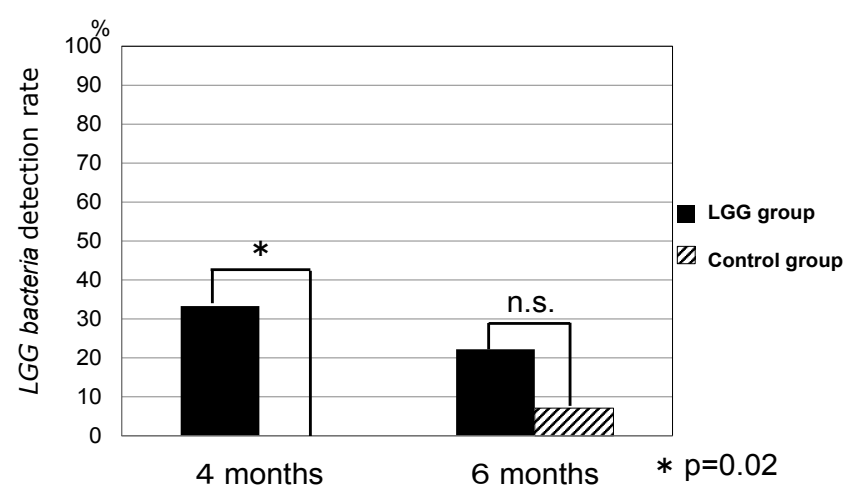

Figure 1: LGG bacteria detection rate; This graph compares the detection rate for LGG bacteria, at 4 and 6 months, between the LGG and the control group, with the detection rate being significantly higher at 4 months of age in the LGG than control group (chi-squared test, $\mathrm{p}=0.02$ ).

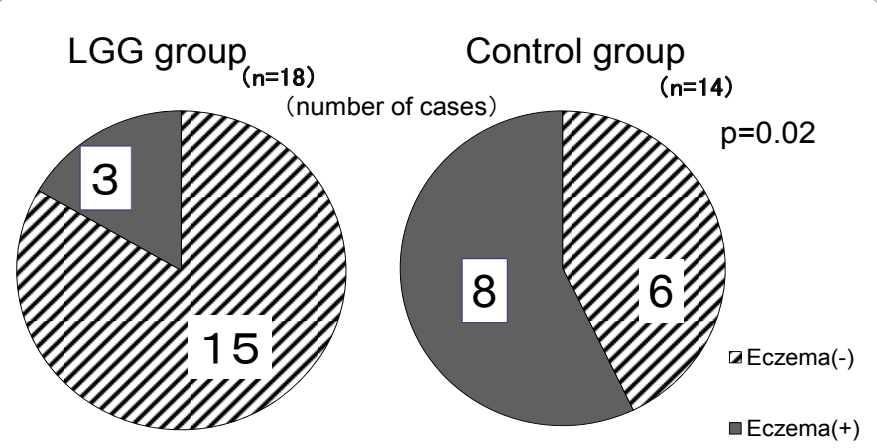

Eczema (+) $16.7 \%$

Eczema(+) $57.1 \%$

Figure 2: Presence of eczema at 1 year of age; Between-group differences in the detection of eczema at 1 year of age, with the rate being significantly lower in the LGG group (16.7\%) than the control group (57.1\%; chi-squared test, $p=0.02$ ).

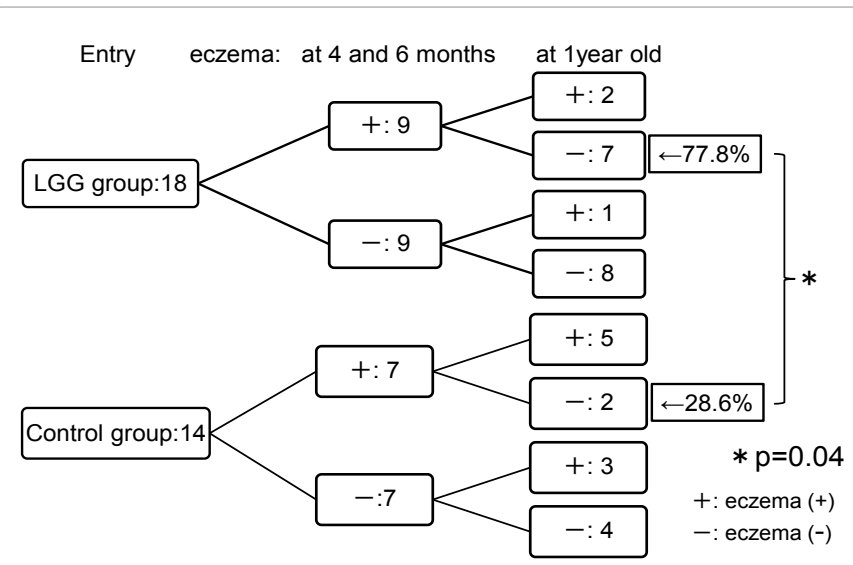

Figure 3: Relationship between the incidence of eczema (at 4 and 6 months) and eczema at 1 year of age between the two groups, with a decrease from 6 months to 1 years of $77.8 \%$ in the LGG group, compared to 28.65 in the control group (chi-squared test, $\mathrm{p}=0.04$ )

Relationship between the presence of LGG bacteria at 4 and 6 months of age and the occurrence of eczema at 1 year of age

We investigated the association between LGG bacteria detected in stool samples at 4 and 6 months of age and the prevalence of eczema at 1 year of age. The prevalence rate of eczema at 1 year of age was lower among infants in whom LGG bacteria were recovered in feces at 4 and/or 6 months of age than in the group of infants in whom LGG bacteria were not detected, although this difference was not significant (Figure 4).

We also compared the detection of rate of LGG bacteria between the two groups and the relationship between LGG bacteria and the prevalence rate of eczema between the two groups at 1 year of age. Regardless of the status of LGG administration to mothers in late gestation, the presence of LGG bacteria was associated with a lower prevalence of eczema at 1 year of age (Figure 5a). Furthermore, we found that the incidence of eczema at 1 year of age was significantly lower in infants in the LGG than in the control group, even when LGG bacteria were not detected at 4 and/or 6 months of age $(\mathrm{p}<0.05$; Figure $5 \mathrm{~b})$.

\section{Relationship between the presence of eczema and LGG administration}

We evaluated the relative association between LGG administration, Lactobacillus and LGG detection and the incidence of eczema at 1 year of age, using logistic regression analysis. The incidence of eczema at 1 year of age was significantly lower in infants born to women who were administered LGG, compared to the control group (Figure 6).

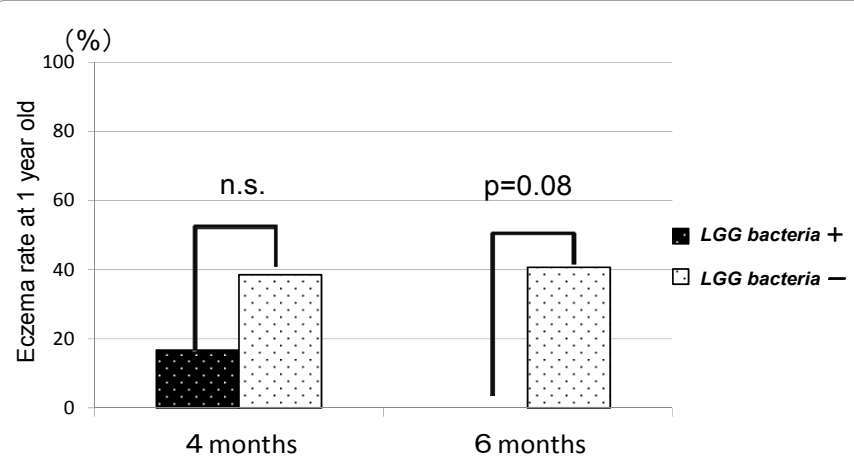

Figure 4: Relationship between detection of LGG bacteria and eczema at year of age.

This figure shows the prevalence of eczema at 1 year as a function of the detection rate of LGG bacteria at 4 and 6 months for all infants $(N=32)$, with the prevalence at 1 year being lower among infants with LGG bacteria, although this difference was not significant (n.s).

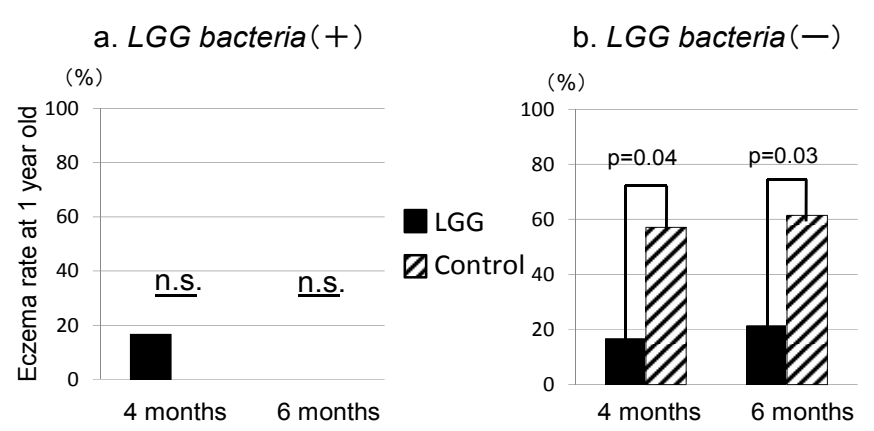

Figure 5: Relationship between the incidence of LGG bacteria and eczema at 1 year of age; a) Cases of eczema at 1 year were lower in infants with LGG bacteria, regardless of group; b) The prevalence of eczema at 1 year was significantly lower in the LGG than the control group, even when LGG bacteria were not detected at $4(p=0.04)$ and $6(p=0.03)$ months. 


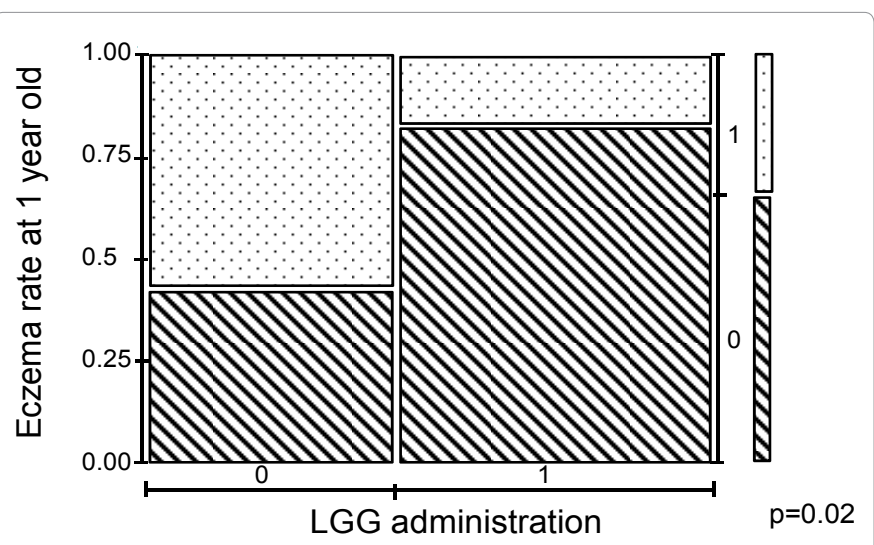

Figure 6: Relationship between the presence of eczema and LGG administration; Logistic regression analysis indicated that the prevalence rate of eczema at 1 year of age was significantly lower after administering LGG to pregnant women $(p=0.02)$.

\section{Discussion}

Our findings support the benefit of providing LGG yogurt to pregnant women during the last month of pregnancy in enhancing the growth of LGG bacteria in the intestinal tract of their newborns before weaning, with an increase in intestinal LGG bacteria being associated with a lower risk of eczema at 1 year of age.

As well, the prevalence rate of eczema at the age of 1 year was significantly lower in the LGG than the control group, even among infants who had eczema at 6 months.

According to the meta-analysis by Panduru et al. [4], specific probiotics may offer protection against atopic diseases if they are administered in the pre- and post-natal period in both the general infant population and in infants at risk for allergies. In this systematic review, however, one study included only prenatal probiotic administration. Moreover, comparison of findings between studies is difficult because of the differences in the type of probiotics administrated, the number of bacteria included in the probiotic preparation, the administration period, and the timing of the assessment of eczema in babies.

There has been continued interest in the protective role of probiotics, with several studies reporting beneficial effects of specific probiotics in lowering the risk for allergy development, and specifically in lowering the incidence rate of eczema in infants [11-16]. The Philippine guidelines on maternal and child health [17] recommend the administration of probiotics to women during pregnancy and lactation. However, although reviews have supported a role of probiotics in preventing eczema in infants, the quality of this evidence is still low [18-21].

In our study, we identified a lower incidence rate of eczema at 1 year of age in the LGG group, regardless of the detection of LGG bacteria at 4 and 6 months. Therefore, it is possible to consider that LGG administration does not only influence the intestinal bacteria of infants after birth, but also exerts a protective effect during the fetal stage.

The lactation period has been reported to be a critical period for allergy development. Recent studies have detected bacterial DNA in the placenta, umbilical cord blood and amniotic fluid [22-26]. Therefore, although the mechanism is still unknown, there is a possibility of a transfer of the intestinal bacteria from the mother to the fetus, via hematogenous and lymphogenous routes, and that such transfer might influence the immune response of the fetus in utero. This fetal immune response could further be enhanced by the prenatal administration of LGG, as in our study.

According to Khan et al. [27] the intra-uterine environment influences allergy development in infants. However, according to the review by West et al., only a few reports have examined the benefits of probiotic administration administered only in the prenatal or postnatal period [20]. Specifically, Rø et al. [28] speculated that the effect of perinatal probiotic administration was mediated via a reduction in the proportion of Th22 cells, which is likely to be the same mechanism mediating the beneficial effects that we identified in our study. This pathway could explain the effectiveness of probiotics in lowering an infant's risk of developing an allergy. We are continuing to explore this avenue through a study of maternal microbiota in specimens obtained from umbilical cord blood, breast milk, intravaginal fluid, and perimammary skin.

In our current study, we administered probiotics to pregnant mothers, and not to their infants. The reason was that, although it is generally said that probiotic administration to infants is safe [29], some studies have reported an increased risk for sepsis associated with probiotic administration [30-33]. Furthermore, with emphasis on maternal safety, mothers were given a probiotic yogurt that had been developed and approved as a commercial FOSHU product.

Our study cohort included infants born vaginally, who received breast milk (exclusive breast feeding) for at least 1 month after birth and who did not use oral antibiotics or steroid ointment up to the age of 6 months. Therefore, our measured effects of LGG were not influenced by the use of antibiotics and steroid medication. The presence of eczema at the age of 1 year was significantly lower in the LGG than control group, which was comparable to previously reported results. We did not restrict the mothers' ingestion of yogurt after birth, which was not different between the two groups. Therefore, the benefits that we identified could be specifically related to the prenatal maternal use of probiotics. However, as the occurrence of eczema was reported by parents, and not diagnosed by a doctor, it was not possible for us to evaluate differences between light and severe eczema, with light eczema not requiring topical steroid treatment. This is a limitation of our trial. In addition, the sample size was small. We plan to investigate by increasing the number of samples in the future.

In summary, LGG administration to pregnant women prevented the onset of eczema in their infants at the age of 1 year. In addition, in the LGG group, the occurrence of eczema was lower than in the control group, even if $L G G$ bacteria were not detected in infant feces. Based on our findings, we propose that maternal LGG administration might influence the microbiota transfer to the infant through the placenta and breast milk. We plan to further investigate the mechanisms of action of LGG administration on the risk for allergy development and eczema more specifically.

\section{Clinical Trial Registration}

UMIN-CTR Clinical Trial Registry (UMIN 000017255)

\section{Acknowledgement}

We thank Dr. Juhani Aakko, Himanshu Kumar Juha-Pekka Pursiheimo in Turku University FFF for suggested data analyses for microbiota.

Thanks also to Hiroyuki Matsuura in Toho University school of medicine for advice on multivariate analysis and statistical processing. 
Citation: Komine Y, Watanabe M, Soutome T, Uchino T, Dobashi M, et al. (2018) Maternal Consumption of Lactobacillus GG During Pregnancy Prevents Infant Eczema at 1 Year of Age. J Allergy Ther 9: 277. doi:10.4172/2155-6121.1000277

Page 5 of 5

\section{References}

1. Kalliomäki M, Salminen S, Arvilommi H, Kero P, Koskinen P, et al. (2001) Probiotics in primary prevention of atopi disease: a randomised placebocontrolled trial. Lancet 357: 1076-1079.

2. Zuccotti G, Meneghin F, Aceti A, Barone G, Callegari ML, et al. (2015) Probiotics for prevention of atopic diseases in infants: systematic review and meta-analysis. Allergy 70: 1356-1371.

3. Cuello-Garcia CA, Brożek JL, Fiocchi A, Pawankar R, Yepes-Nuñez JJ, et al. (2015) Probiotics for the prevention of allergy: A systematic review and metaanalysis of randomized controlled trials. Allergy Clin Immunol 136: 952-961.

4. Panduru M, Panduru NM, Sălăvăstru CM, Tiplica GS (2015) Probiotics and primary prevention of atopic dermatitis: a meta-analysis of randomized controlled studies. Eur Acad Dermatol Venereol 29: 232-242.

5. Fiocchi A, Pawankar R, Cuello-Garcia C, Ahn K, Al-Hammadi S, et al. (2015) World Allergy Organization: McMaster University Guidelines for Allergic Disease Prevention (GLAD-P): Probiotics. World Allergy Organ J 8: 4

6. Komine Y, Watanabe M, Soutome T, Uchino K, Uchino T, et al. (2014) Maternal consumption of Lactobacillus rhamnosus GG yogurt during pregnancy promotes bifidobacteria growth in intestinal microflora of infants. J Med Soc Toho 61: 3-12.

7. Stalder JF, Taïeb A, Atherton DJ, Bieber P, Bonifazi E, et al. (1993) Severity scoring of atopic dermatitis: The SCORAD index: Consensus report of the european task force on atopic dermatitis. Dermatology 186: 23- 31.

8. Kozich JJ, Westcott SL, Baxter NT, Sarah K (2013) Development of a dualindex sequencing strategy and curation pipeline for analyzing amplicon sequence data on the MiSeq Illumina Sequencing Platform James. App Environ Microbiol 79: 5112-5120.

9. Brandt K, Alatossava T (2003) Specific identification of certain probiotic Lactobacillus rhamnosus strains with PCR primers based on phagerelated sequences. Int J Food Microbiol 84: 189-196.

10. Salminen S, Endo A, Isolauri E, Scalabrin D (2016) Early gut colonization with Lactobacilli and Staphylococcus in infants: The hygiene hypothesis extended. J Pediatr Gastroenterol Nutr 62: 80-86.

11. Barthow C, Wickens K, Stanley T, Mitchell EA, Maude R, et al. (2016) The probiotics in pregnancy study (PiP study): Rationale and design of a doubleblind randomised controlled trial to improve maternal health during pregnancy and prevent infant eczema and allergy. BMC Pregnancy Childbirth 16: 133.

12. Simpson MR, Dotterud CK, Storrø O, Johnsen R, Øien T (2015) Perinata probiotic supplementation in the prevention of allergy related disease: 6 year follow up of a randomised controlled trial. BMC Dermatol 15: 13

13. Awasthi S, Wilken R, Patel F, German JB, Mills DA, et al. (2016) Dietary supplementation with Bifidobacterium longum subsp. infantis (B. infantis) in healthy breastfed infants: Study protocol for a randomised controlled trial. Trials 17: 340 .

14. Peldan P, Kukkonen AK, Savilahti E, Kuitunen M (2017) Perinatal probiotics decreased eczema up to 10 years of age, but at 5-10 years, allergic rhinoconjunctivitis was increased. Clin Exp Allergy 47: 975-979.

15. Lundelin K, Poussa T, Salminen S, Isolauri E (2017) Long-term safety and efficacy of perinatal probiotic intervention: Evidence from a follow-up study of four randomized, double-blind, placebo-controlled trials. Pediatr Allergy Immunol 28: 170-175.
16. Damm JA, Smith B, Greisen G, Krogfelt KA, Clausen ML, et al. (2017) The influence of probiotics for preterm neonates on the incidence of atopic dermatitisresults from a historically controlled cohort study. Arch Dermatol Res 309: 259-264.

17. Recto MST, Genuino MLG, Castor MAR, Casis-Hao RJ, Tamondong-Lachica DR, et al. (2017) Dietary primary prevention of allergic diseases in children: the Philippine guidelines. Asia Pac Allergy 7: 102-114.

18. Forsberg A, West CE, Prescott SL, Jenmalm MC (2016) Pre- and probiotics for allergy prevention: time to revisit recommendations? Clin Exp Allergy 46: 1506-1521.

19. Zhang GQ, Hu HJ, Liu CY, Zhang Q, Shakya S, et al. (2016) Probiotics for prevention of atopy and food hypersensitivity in early childhood: A PRISMAcompliant systematic review and meta-analysis of randomized controlled trials. Medicine (Baltimore) 95: e2562.

20. West CE, Jenmalm MC, Kozyrskyj AL, Prescott SL (2016) Probiotics for treatment and primary prevention of allergic diseases and asthma: looking back and moving forward. Expert Rev Clin Immunol 12: 625-639.

21. Friedrich AD, Paz ML, Leoni J, González Maglio DH (2017) Message in a bottle: Dialog between intestine and skin modulated by probiotics. Int $\mathrm{J}$ Mol Sci 18: E1067

22. Rautava S, Collado MC, Salminen S, Isolauri E (2012) Probiotics modulate host-microbe interaction in the placenta and fetal gut: a randomized, doubleblind, placebo-controlled trial. Neonatology 102: 178-184.

23. Aagaard K, Ma J, Antony KM, Ganu R, Petrosino J, et al. (2014) The placenta harbors a unique microbiome. Sci Transl Med 6: 237ra65

24. Funkhouser LJ, Bordenstein SR (2013) Mom knows best: the universality of maternal microbial transmission. PLoS Biol 11: e1001631.

25. Stout MJ, Conlon B, Landeau M, Lee I, Bower C, et al. (2013) Identification of intracellular bacteria in the basal plate of the human placenta in term and preterm gestations. Am J Obstet Gynecol 208: 226.e1-7.

26. Abrahamsson TR, Wu RY, Jenmalm MC (2015) Gut microbiota and allergy: The importance of the pregnancy period. Pediatr Res 77: 214-219.

27. Khan TK, Palmer DJ, Prescott SL (2015) In-utero exposures and the evolving epidemiology of paediatric allergy. Curr Opin Allergy Clin Immunol 15: 402-408.

28. Rø ADB, Simpson MR, Rø TB, Storrø O, Johnsen R, et al. (2017) Reduced Th22 cell proportion and prevention of atopic dermatitis in infants following maternal probiotic supplementation. Clin Exp Allergy 47: 1014-1021.

29. Salminen S, Endo A, Isolauri E, Scalabrin D (2016) Early gut colonization with Lactobacilli and Staphylococcus in infants: The hygiene hypothesis extended. JPGN 62: 80-86.

30. Mackay AD, Taylor MB, Kibbler CC, Hamilton-Miller JM (1999) Lactobacillus endocarditis caused by a probiotic organism. Clin Microbiol Infect 5: 290-292.

31. Salminen MK, Tynkkynen S, Rautelin H, Saxelin M, Vaara M, et al. (2002) Lactobacillus bacteremia during a rapid increase in probiotic use of Lactobacillus rhamnosus GG in Finland. Clin Infect Dis 35: 1155-1160.

32. Ohishi A, Takahashi S, Ito Y, Ohishi Y, Tsukamoto K, et al. (2010) Bifidobacterium septicemia associated with postoperative probiotic therapy in a neonate with omphalocele. J Pediatr 156: 679-681.

33. Zbinden A, Zbinden R, Berger C, Arlettaz R (2015) Case series of Bifidobacterium longum bacteremia in three preterm infants on probiotic therapy. Neonatol 107 $56-59$ 